\title{
Enhanced thermally aided memory performance using few-layer ReS2 transistors
}

Goyal, Natasha; MacKenzie, David M.A.; Panchal, Vishal; Jawa, Himani; Kazakova, Olga; Petersen, Dirch Hjorth; Lodha, Saurabh

Published in:

Applied Physics Letters

Link to article, DOI:

$10.1063 / 1.5126809$

Publication date:

2020

Document Version

Publisher's PDF, also known as Version of record

Link back to DTU Orbit

Citation (APA):

Goyal, N., MacKenzie, D. M. A., Panchal, V., Jawa, H., Kazakova, O., Petersen, D. H., \& Lodha, S. (2020). Enhanced thermally aided memory performance using few-layer ReS ${ }_{2}$ transistors. Applied Physics Letters, 116(5), [052104]. https://doi.org/10.1063/1.5126809

\section{General rights}

Copyright and moral rights for the publications made accessible in the public portal are retained by the authors and/or other copyright owners and it is a condition of accessing publications that users recognise and abide by the legal requirements associated with these rights.

- Users may download and print one copy of any publication from the public portal for the purpose of private study or research.

- You may not further distribute the material or use it for any profit-making activity or commercial gain

- You may freely distribute the URL identifying the publication in the public portal 


\section{Enhanced thermally aided memory performance using few-layer $\mathrm{Re}_{2}$ transistors}

Cite as: Appl. Phys. Lett. 116, 052104 (2020); https://doi.org/10.1063/1.5126809

Submitted: 17 September 2019 . Accepted: 19 January 2020 . Published Online: 06 February 2020

Natasha Goyal (D), David M. A. Mackenzie (D), Vishal Panchal, Himani Jawa, Olga Kazakova (D), Dirch Hjorth Petersen, and Saurabh Lodha

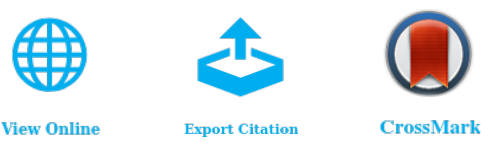

\section{ARTICLES YOU MAY BE INTERESTED IN}

Interfacial Dzyaloshinskii-Moriya interaction between ferromagnetic insulator and heavy metal

Applied Physics Letters 116, 052404 (2020); https://doi.org/10.1063/1.5134762

Solid-state cooling by stress: A perspective

Applied Physics Letters 116, 050501 (2020); https://doi.org/10.1063/1.5140555

Contactless pick-and-place of millimetric objects using inverted near-field acoustic levitation Applied Physics Letters 116, 054104 (2020); https://doi.org/10.1063/1.5138598

\section{Lock-in Amplifiers up to $600 \mathrm{MHz}$}
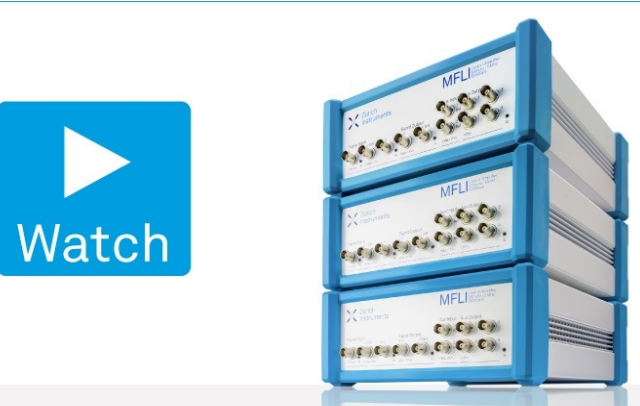


\title{
Enhanced thermally aided memory performance using few-layer $\operatorname{ReS}_{2}$ transistors
}

\author{
Cite as: Appl. Phys. Lett. 116, 052104 (2020); doi: 10.1063/1.5126809 \\ Submitted: 17 September 2019 • Accepted: 19 January 2020 . \\ Published Online: 6 February 2020
}

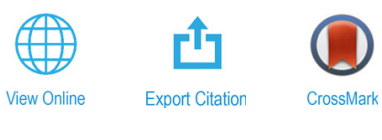

Natasha Goyal, ${ }^{1}$ (D) David M. A. Mackenzie, ${ }^{2}$ iD Vishal Panchal, ${ }^{3}$ Himani Jawa, ${ }^{1}$ Olga Kazakova, ${ }^{4}$ Dirch Hjorth Petersen, ${ }^{5}$ and Saurabh Lodha,

\begin{abstract}
AFFILIATIONS
${ }^{7}$ Department of Electrical Engineering, Indian Institute of Technology Bombay, Mumbai 400076, India ${ }^{2}$ Department of Electronics and Nanoengineering, Aalto University, PO Box 13500, FI-00076 Aalto, Finland

${ }^{3}$ Time, Quantum and Electromagnetics division, National Physical Laboratory, Teddington, Middlesex TW11 OLW, United Kingdom

${ }^{4}$ Quantum Technology, National Physical Laboratory, Teddington, Middlesex TW11 OLW, United Kingdom

${ }^{5}$ Department of Physics, Technical University of Denmark, Fysikvej, Building 311, DK-2800 Kgs. Lyngby, Denmark

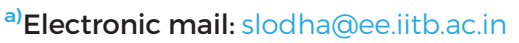

\begin{abstract}
Thermally varying hysteretic gate operation in few-layer $\mathrm{ReS}_{2}$ and $\mathrm{MoS}_{2}$ back gate field effect transistors (FETs) is studied and compared for memory applications. Clockwise hysteresis at room temperature and anti-clockwise hysteresis at higher temperature $\left(373 \mathrm{~K}\right.$ for $\mathrm{ReS}_{2}$ and $400 \mathrm{~K}$ for $\mathrm{MoS}_{2}$ ) are accompanied by step-like jumps in transfer curves for both forward and reverse voltage sweeps. Hence, a steplike conductance (STC) crossover hysteresis between the transfer curves for the two sweeps is observed at high temperature. Furthermore, memory parameters such as the RESET-to-WRITE window and READ window are defined and compared for clockwise

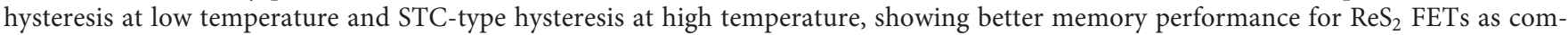
pared to $\mathrm{MoS}_{2}$ FETs. Smaller operating temperature and voltage along with larger READ and RESET-to-WRITE windows make ReS 2 FETs a better choice for thermally aided memory applications. Finally, temperature dependent Kelvin probe force microscopy measurements show decreasing (constant) surface potential with increasing temperature for $\operatorname{ReS}_{2}\left(\mathrm{MoS}_{2}\right)$. This indicates less effective intrinsic trapping at high temperature in $\mathrm{ReS}_{2}$, leading to earlier occurrence of STC-type hysteresis in $\mathrm{ReS}_{2}$ FETs as compared to MoS 2 FETs with increasing temperature.
\end{abstract}

Published under license by AIP Publishing. https://doi.org/10.1063/1.5126809

Recently, two-dimensional (2D) layered materials have attracted significant research interest for memory applications. Thermally assisted non-volatile memories (NVMs) have been demonstrated using monolayer and few-layer $\mathrm{MoS}_{2}{ }^{1,2}$ In several other reports, large hysteresis in transistor transfer curves, normally undesirable for device performance, has been utilized for NVM applications. ${ }^{3-6}$ All of these reports use $\mathrm{MoS}_{2}$ as the channel or as the charge trapping layer. In thermally assisted NVMs, locally generated heat is exploited for switching between different memory states. $\mathrm{MoS}_{2}$ has shown excellent switching characteristics compared to other transition metal dichalcogenides (TMDs). ${ }^{7}$ Low off current $\left(\mathrm{I}_{\text {off }}\right)$ and a high on/off current $\left(\mathrm{I}_{o n} / \mathrm{I}_{\text {off }}\right)$ ratio (due to a large bandgap) along with a low sub-threshold slope and high effective mass are some of the advantages of $\mathrm{MoS}_{2}$, which make it a desirable switching material for memory applications. ${ }^{8,9}$ Among the TMDs, $\mathrm{ReS}_{2}$ has also garnered significant attention recently ${ }^{10,11}$ since it behaves as decoupled monolayers stacked on top of each other due to the lack of interlayer coupling and weak interlayer registry. ${ }^{12}$ Hence, $\mathrm{ReS}_{2}$ remains a direct bandgap semiconductor $\left(\mathrm{E}_{G}=1.5 \mathrm{eV}\right)$ from monolayer to bulk, showing no direct to indirect bandgap crossover as is shown by other TMDs, making it a preferred material for optoelectronic applications. ${ }^{10,13}$

With the increasing packing density of field effect transistors (FETs) on a single wafer, high performance ICs can reach an operating temperature (T) of 370-530 K (Ref. 14), making it important to understand and exploit the changes that occur in the properties of 2D materials at high $\mathrm{T}$. Thermally assisted memory is one such application where locally generated heat is exploited to aid the switching between RESET (RST/STATE 0) and WRITE (WR/STATE 1) states. ${ }^{15}$ It can enable embedded in-memory computing that has emerged as a key hardware bottleneck for artificial intelligence/machine learning technologies. However, in-memory computing requires more computational power per unit volume of data storage in the RAM and parallel 
TABLE I. Comparison of memory parameters obtained in this work with the previous reports on thermally assisted memory using MoS $\mathrm{S}_{2}$ as the channel material.

\begin{tabular}{|c|c|c|c|c|c|c|}
\hline References & Material & $\begin{array}{l}\text { Operating } \\
\text { temperature }\end{array}$ & $\begin{array}{l}\text { Operating voltage } \\
\qquad\left(\mathrm{V}_{p-p}\right)\end{array}$ & $\begin{array}{l}\text { RST-to-WR window } \\
\quad\left(\Delta \mathrm{V}_{t h} / \mathrm{V}_{p-p}\right)\end{array}$ & $\mathrm{RD}$ window & Hysteresis type \\
\hline 1 & Monolayer, $\mathrm{MoS}_{2}$ & $490 \mathrm{~K}$ & $-40 \mathrm{~V}$ to $+40 \mathrm{~V}$ & 0.44 & 5.5 & $\mathrm{STC}+\mathrm{ACW}$ \\
\hline 21 & Monolayer, $\mathrm{MoS}_{2}$ & $350 \mathrm{~K}$ & $-30 \mathrm{~V}$ to $+15 \mathrm{~V}$ & 0.50 & 7 & CW \\
\hline 22 & Multilayer $\mathrm{MoS}_{2}$ & $300 \mathrm{~K}$ & $-30 \mathrm{~V}$ to $+30 \mathrm{~V}$ & 0.1 & - & CW \\
\hline This work & Few-layer $\mathrm{MoS}_{2}$ & $400 \mathrm{~K}$ & $-100 \mathrm{~V}$ to $+60 \mathrm{~V}$ & 0.16 & 1.9 & $\mathrm{STC}+\mathrm{ACW}$ \\
\hline This Work & Few-layer $\mathrm{ReS}_{2}$ & $375 \mathrm{~K}$ & $-100 \mathrm{~V}$ to $+30 \mathrm{~V}$ & 0.58 & 7.4 & $\mathrm{STC}+\mathrm{ACW}$ \\
\hline
\end{tabular}

distributed processing. This increases the operational $\mathrm{T}$ of data centers, resulting in several reliability concerns. ${ }^{16,17}$ Therefore, enabling low $\mathrm{T}$ memory operation in $2 \mathrm{D}$ materials is timely and relevant. In this study, thermally varying hysteretic gate operation, in few-layer $\mathrm{MoS}_{2}$ and in few-layer $\mathrm{ReS}_{2}$, is studied and compared for memory applications. Four-terminal back gate FETs are used in this study to eliminate the contribution from contact resistance. ${ }^{18}$ Clockwise (CW) hysteresis is observed for both $\mathrm{ReS}_{2}$ and $\mathrm{MoS}_{2}$ at room temperature (RT), whereas anti-clockwise (ACW) hysteresis along with step-like jumps in the transfer curves leading to a conductance crossover between the forward sweep (FS, $-100 \mathrm{~V}$ to $+100 \mathrm{~V}$ ) and reverse sweep (RS, $+100 \mathrm{~V}$ to $-100 \mathrm{~V}$ ) directions (step-like conductance crossover hysteresis or STC hysteresis) is observed at high T. A similar behavior has been previously reported for monolayer $\mathrm{MoS}_{2}$ FETs. ${ }^{1}$ Here, we observe this behavior in both few-layer $\mathrm{MoS}_{2}$ at $400 \mathrm{~K}$ and few-layer $\mathrm{ReS}_{2}$ at $375 \mathrm{~K}$, attributing the RT CW hysteresis to the dominance of native intrinsic traps and the conductance crossover at high $\mathrm{T}$ to charge exchange between the $\mathrm{p}^{+} \mathrm{Si}$ back gate and gate oxide $\mathrm{SiO}_{2}$. The charge can be trapped in the oxide near the $2 \mathrm{D}$ channel/dielectric or the back gate/dielectric interface. ${ }^{1,19,20}$ However, the latter is found to be dominating at high $\mathrm{T}$ in these systems. ${ }^{2} \mathrm{~A}$ comparison of this work with previous reports on thermally assisted memory is presented in Table I.

We observe lower operating voltage $\left(\mathrm{V}_{p-p}\right)$, a larger RST-to-WR window, defined as $\Delta \mathrm{V}_{t h} / \mathrm{V}_{p-p}$ (where $\Delta \mathrm{V}_{\text {th }}$ is the hysteresis width), and a larger READ (RD) window for STC hysteresis in $\mathrm{ReS}_{2}$ devices. Improved memory parameters for $\mathrm{ReS}_{2}$ FETs at much lower $\mathrm{T}$ are attributed to a rapidly reducing effect of intrinsic traps with increasing $\mathrm{T}$. This is verified by T-dependent Kelvin probe force microscopy (KPFM) measurements, which indicate the decreasing work function for $\operatorname{ReS}_{2}\left(\Phi_{\text {ReS }_{2}}\right)$ unlike an almost constant work function for $\mathrm{MoS}_{2}$ $\left(\Phi_{\mathrm{MoS}_{2}}\right)$ with increasing T.

Figure 1(a) shows an optical image (top) and a 3D schematic (bottom) of the four-terminal back gated transistor structure used to study thermally varying hysteretic gate operation for both $\mathrm{MoS}_{2}$ and $\mathrm{ReS}_{2}$ as the channel materials. The TMD sheets were mechanically exfoliated (using adhesive blue tape) from bulk crystals on heavily doped p-type silicon substrates with a $280 \mathrm{~nm} \mathrm{SiO}_{2}$ layer on top. ${ }^{23,24}$ Optical microscopy was used to identify the flakes for further processing. Source/drain electrodes were then patterned using electron beam lithography followed by metal deposition. $10 \mathrm{~nm} \mathrm{Cr}$ and $100 \mathrm{~nm} \mathrm{Au}$ were used to form source/drain metal contacts with the flakes.

Figures 1 and 2 show the conductance $(G)$ vs back gate voltage $\left(V_{G S}\right)$ curves for varying T. Figures $1(d)$ and 2(d) define the RST, RD, and WR operations for the $\mathrm{ReS}_{2}$ and $\mathrm{MoS}_{2}$ memories, respectively.
All the measurements reported in this work start with the FS followed by RS. We observe two significant step-like jumps in the $\mathrm{G}-\mathrm{V}_{G S}$ plots at higher T. The first one occurs during FS at $20 \mathrm{~V}$ for $\mathrm{ReS}_{2}$ [Fig. 1(d)] and at $35 \mathrm{~V}$ [Fig. 2(d)] for $\mathrm{MoS}_{2}$. The second one occurs during RS at $-76 \mathrm{~V}$ for $\mathrm{ReS}_{2}$ [Fig. 1(d)] and $-66 \mathrm{~V}$ for $\mathrm{MoS}_{2}$ [Fig. 2(d)]. As a result STC hysteresis emerges at $373 \mathrm{~K}$ and $400 \mathrm{~K}$ for $\mathrm{ReS}_{2}$ and $\mathrm{MoS}_{2}$, respectively. These jumps can be prominently observed in the transconductance $\left(\mathrm{g}_{m}\right)$ curves in Figs. S1(a) and S1(b) of the supplementary material for $\mathrm{ReS}_{2}$ and $\mathrm{MoS}_{2}$, respectively. Along with the jumps occurring at higher T, a switch from CW hysteresis at RT to ACW hysteresis at higher $\mathrm{T}$ can also be observed in the transfer curves for both $\mathrm{ReS}_{2}$ and $\mathrm{MoS}_{2}$.

The changing hysteresis behavior with varying $\mathrm{T}$ is shown in Figs. 3(a) and $3(\mathrm{~b})$ via the change in threshold voltage $\left(\mathrm{V}_{\text {th }}\right)$ for FS (STATE 1, $\mathrm{V}_{t h}^{F S}$ ) and RS (STATE 0, $\mathrm{V}_{t h}^{R S}$ ) with T for $\mathrm{MoS}_{2}$ and $\mathrm{ReS}_{2}$, respectively. A transition from $C W\left(\mathrm{~V}_{t h}^{F S}<\mathrm{V}_{t h}^{R S}\right)$ to ACW $\left(\mathrm{V}_{t h}^{F S}>\mathrm{V}_{t h}^{R S}\right)$ hysteresis with increasing $\mathrm{T}$ can be observed. A larger hysteresis width $\left(\Delta \mathrm{V}_{t h}=\mathrm{V}_{t h}^{R S}-\mathrm{V}_{t h}^{F S}\right)$ for $\mathrm{ReS}_{2}$ at much lower $\mathrm{T}$ compared to $\mathrm{MoS}_{2}$ can also be seen. As marked in Figs. 1(d) and 2(d), WR and RST operations are carried out at the end of FS and RS, respectively. A larger $\Delta \mathrm{V}_{\text {th }}$ implies a larger RST-to-WR window, a desirable feature for
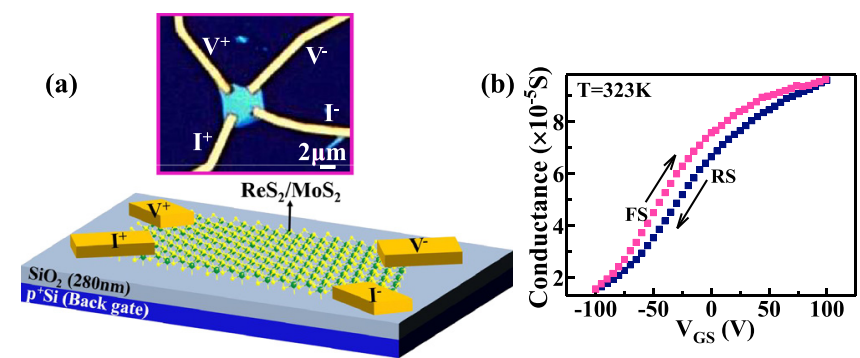

(c)

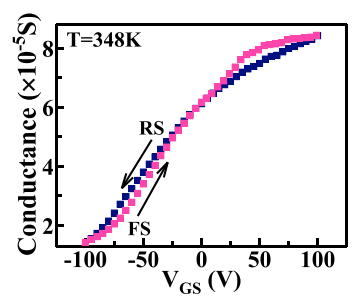

(d)

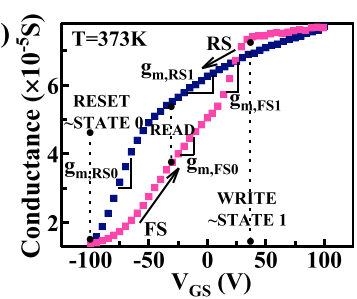

FIG. 1. (a) Optical image (top) and schematic (bottom) of back-gated vdP FETs with the $\mathrm{ReS}_{2} / \mathrm{MoS}_{2}$ channel. $\mathrm{G}(\mathrm{S})$ vs $\mathrm{V}_{\mathrm{GS}}(\mathrm{V})$ at $\mathrm{V}_{d}=2 \mathrm{~V}$ for $\mathrm{ReS}_{2}$ FET showing the evolving hysteresis width with increasing $\mathrm{T}$ at (b) $323 \mathrm{~K}$, (c) $348 \mathrm{~K}$, and (d) $373 \mathrm{~K}$, depicting STATES 0 and 1 corresponding to WRITE (WR) and RESET (RST), respectively. 

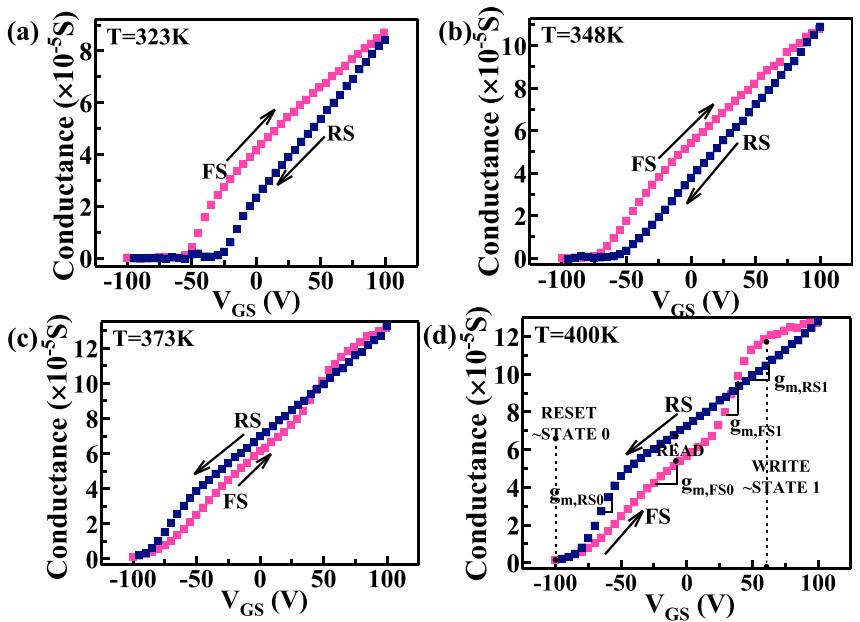

FIG. 2. $\mathrm{G}(\mathrm{S})$ vs $\mathrm{V}_{\mathrm{GS}}(\mathrm{V})$ at $\mathrm{V}_{d}=0.5 \mathrm{~V}$ for $\mathrm{MoS}_{2}$ FET showing the evolving hysteresis width with increasing $T$ at (a) $323 \mathrm{~K}$, (b) $348 \mathrm{~K}$, (c) $373 \mathrm{~K}$, and (d) $400 \mathrm{~K}$, indicating memory STATE 0 and STATE 1.

memory operation. Hence, a larger RST-to-WR window is observed for $\mathrm{ReS}_{2}$ as compared to $\mathrm{MoS}_{2}$. Figure 3(c) shows the ratio of $\mathrm{g}_{m}$ closer to STATE 0 ( $g_{m, R S 0}$ for RS and $g_{m, F S 0}$ for FS) to $g_{m}$ closer to STATE 1 ( $\mathrm{g}_{m, R S 1}$ for RS and $\mathrm{g}_{m, F S 1}$ for FS) as marked in Figs. 1(d) and 2(d). The difference between $\mathrm{g}_{m, R S 0} / \mathrm{g}_{m, R S 1}$ and $\mathrm{g}_{m, F S 0} / \mathrm{g}_{m, F S 1}$ is defined as the $\mathrm{RD}$ window, which rapidly increases with increasing $\mathrm{T}$ for $\mathrm{ReS}_{2}$ at much lower $\mathrm{T}$ as compared to $\mathrm{MoS}_{2}$.

When $\mathrm{T}$ is increased from RT to higher $\mathrm{T}$, a change from $\mathrm{CW}$ to ACW hysteresis along with step-like jumps occurs during both FS
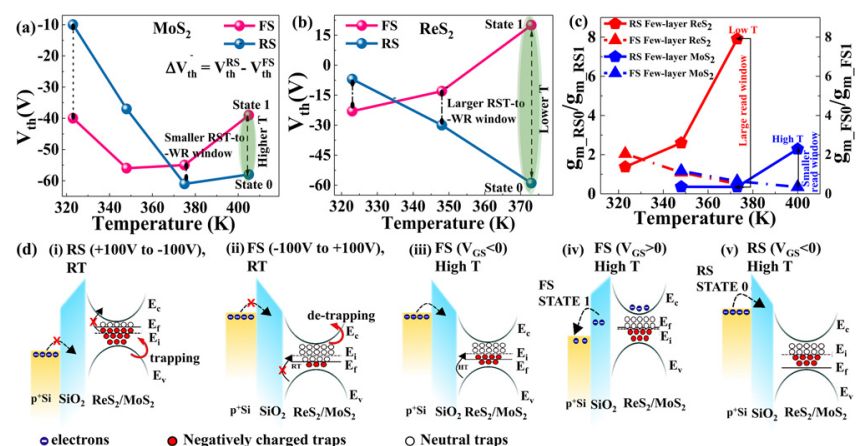

FIG. 3. The change in $V_{t h}^{F S}$ and $V_{t h}^{R S}$ with varying $T$ for (a) $M_{0} S_{2}$ and (b) ReS $S_{2}$. The shaded green region shows a larger RST-to-WR window for $\mathrm{ReS}_{2}$ as compared to $\mathrm{MoS}_{2}$, at much lower T. (c) $g_{m}$ ratios for $\mathrm{ReS}_{2}$ and $\mathrm{MoS}_{2}$ devices during FS and RS close to STATE 0 and STATE $1 .^{25}$ (d) Band diagrams of the back-gated FETs with $\mathrm{ReS}_{2} / \mathrm{MoS}_{2}$ as the channel material. At RT, (i) the onset of RS moves the Fermi level $\left(E_{f}\right)$ closer to conduction band minimum $\left(E_{c}\right)$, which leads to electron $\left(\mathrm{e}^{-}\right)$trapping below $\mathrm{E}_{f}$ (shown by the solid red arrow), while (ii) the onset of FS causes the de-trapping of $e^{-}$that are above $E_{f}$. As $T$ increases, the intrinsic traps can be occupied by $\mathrm{e}^{-}$at all voltages due to the availability of thermionic energy. Therefore, at higher $\mathrm{T}$, during $\mathrm{FS}$, (iii) when $\mathrm{V}_{G S}<0, \mathrm{e}^{-}$from the $\mathrm{p}^{+}$-Si gate jump into the oxide (dashed arrow) screening the applied $V_{G S}$, while (iv) for $V_{G S}>0$, these $\mathrm{e}^{-}$jump back to the gate, causing the first $g_{m}$ jump (STATE 1). (v) During RS when $V_{G S}<0, e^{-}$from the gate jump again to the oxide re-screening $V_{G S}$, resulting in the occurrence of the second $g_{m}$ jump (STATE 0 ). (leading to STATE 1) and RS (leading to STATE 0). We attribute the fading away of the CW hysteresis with increasing T to electron $\left(\mathrm{e}^{-}\right)$ trapping and de-trapping into the deep level channel traps that occur due to intrinsic defects in materials, as previously reported for fully depleted channels. ${ }^{2,26-28}$ At the onset of RS $(+100 \mathrm{~V})$, these traps below the Fermi level $\left(\mathrm{E}_{f}\right)$ get filled by $\mathrm{e}^{-}$, and hence, an increase in $\mathrm{V}_{\text {th }}$ is observed [Fig. 3(d)-(i)], whereas at the onset of FS $(-100 \mathrm{~V})$, the bands closer to the $\mathrm{SiO}_{2} /$ channel interface bend to favor $\mathrm{e}^{-}$ de-trapping from the intrinsic traps, resulting in reduced $\mathrm{V}_{\text {th }}$ [Fig. 3(d)-(ii)]. As a result, CW hysteresis is seen at RT. At higher T, the deep level traps have an equal probability of getting trapped irrespective of the voltage applied, and hence, the hysteresis starts to fade away. ${ }^{2}$ Instead, an ACW hysteresis with step-like jumps in the G vs $\mathrm{V}_{G S}$ profile starts occurring (at $348 \mathrm{~K}$ for $\mathrm{ReS}_{2}$ and at $373 \mathrm{~K}$ for $\mathrm{MoS}_{2}$ ), which is attributed to the trapping and de-trapping of $\mathrm{e}^{-}$from the gate into the oxide and vice versa with enough activation energy $\left(\mathrm{E}_{A}\right)$ at higher temperature. ${ }^{1}$ At high $\mathrm{T}$, at the beginning of FS $\left(\mathrm{V}_{G S}<0\right)$, the bands bend as shown by the schematic in Fig. 3(d)-(iii) favoring $\mathrm{e}^{-}$injection from the gate into the oxide causing gate field screening. The stored $\mathrm{e}^{-}$give rise to a repulsive field in addition to the gate field as long as $\mathrm{V}_{G S}<0$. For $\mathrm{V}_{G S}>0$, the bands evolve [Fig. 3(d)-(iv)] allowing the trapped oxide $\mathrm{e}^{-}$to jump back into the gate, resulting in a sudden increase in the attractive field seen by the channel and the first $g_{m}$ jump is observed, which leads to STATE 1 of the memory operation during FS. For $\mathrm{V}_{G S}<0$ during RS, the ejection of $\mathrm{e}^{-}$from the oxide into the gate is again favored [Fig. 3(d)-(v)], and we see the second $g_{m}$ jump, leading to STATE 0 of the memory operation. It is important to note that at lower $\mathrm{T}$, the gate $\mathrm{e}^{-}$do not have the $\mathrm{E}_{A}$ required to overcome the barrier between the gate and the oxide. Hence, intrinsic trapping is the dominating mechanism responsible for CW hysteresis at lower $\mathrm{T}$.

Figure 4(a) plots $\Delta \mathrm{V}_{\text {th }}$ (left axis) and trap density $\left(\Delta \mathrm{N}_{T}\right.$, right axis) vs T, extracted from Figs. 1 and 2 for $\mathrm{ReS}_{2}$ (red line) and $\mathrm{MoS}_{2}$ (green line), respectively, using the following equation:

$$
\Delta N_{T}=\frac{\Delta V_{t h} \times C_{o x}}{q},
$$

where the gate oxide capacitance per unit area is given by $\mathrm{C}_{o x}=\frac{\varepsilon_{0} \varepsilon_{r}}{d}, \varepsilon_{r}$ $=3.9$, and oxide thickness $d=280 \mathrm{~nm}$. The fits (dashed lines) to $\Delta \mathrm{V}_{\text {th }}$ and $\Delta \mathrm{N}_{T}$ in Fig. 4(a) are used to extract the oxide trap density $\left(\Delta \mathrm{N}_{O T}\right)$ and intrinsic trap density $\left(\Delta \mathrm{N}_{I T}\right)$ components for $\operatorname{ReS}_{2}$ and $\mathrm{MoS}_{2}$ in Fig. 4(b). $\Delta \mathrm{N}_{O T}$ is considered to be negligible at $\mathrm{RT}$ and the same for both $\mathrm{ReS}_{2}$ and $\mathrm{MoS}_{2}$ at all $\mathrm{T}$ since both types of devices are fabricated on identical $\mathrm{p}^{+} \mathrm{Si} / \mathrm{SiO}_{2}$ substrates. The algebraic sum of $\Delta \mathrm{N}_{O T}$ and $\Delta \mathrm{N}_{I T}$ results in total $\Delta \mathrm{N}_{T}$. For both materials, hysteresis becomes zero at higher $\mathrm{T}$ due to the reducing impact of $\Delta \mathrm{N}_{I T}$, but the effect of $\Delta \mathrm{N}_{I T, R e S_{2}}$ dies faster than $\Delta \mathrm{N}_{I T, M o S_{2}}$. This is explained by a schematic shown in Fig. 4(c), depicting the inversion from CW to ACW hysteresis with increasing $\mathrm{T}$ due to the dominance of $\Delta \mathrm{N}_{I T}$ and $\Delta \mathrm{N}_{O T}$ independently at RT and high $\mathrm{T}$, respectively. The evolution of hysteresis with $\mathrm{T}$ due to only $\Delta \mathrm{N}_{O T}$ is shown by the solid pink line, depicting a negligible hysteresis at lower $\mathrm{T}$, which eventually increases for higher T. However, the solid red and blue lines show the effect of $\Delta \mathrm{N}_{I T}$ on hysteresis for $\mathrm{ReS}_{2}$ and $\mathrm{MoS}_{2}$ with $\mathrm{T}$, respectively. Hence, adding the hysteresis effects from the two kinds of traps gives the net hysteresis change as shown by the dashed lines in Fig. 4(b). Steeper reduction in $\Delta \mathrm{N}_{I T, R e S_{2}}$ with $\mathrm{T}$ as compared to $\Delta \mathrm{N}_{I T, M o S_{2}}$ can be observed. 


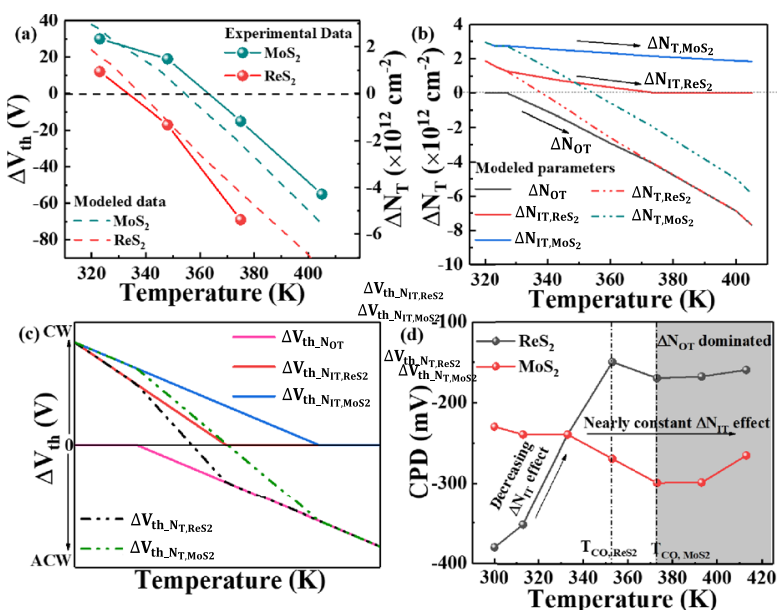

FIG. 4. (a) $\Delta \mathrm{V}_{\text {th }}$ (left axis) and $\Delta \mathrm{N}_{T}$ (right axis) vs $T$ extracted from the experimental data (solid line) along with the fitted data (dashed lines) for $\mathrm{MoS}_{2}$ and $\mathrm{ReS}_{2}$. (b) Evolution of fitted $\Delta \mathrm{N}_{T}$ data (dashed lines) along with $\Delta \mathrm{N}_{O T}, \Delta \mathrm{N}_{\text {IT ReS }}$, and $\Delta \mathrm{N}_{I T, M o S_{2}}$ constituents with $\mathrm{T}$. (b) Schematic explaining the evolution of $\Delta \mathrm{V}_{\text {th }}$ due to $\Delta \mathrm{V}_{\text {th, } N_{\text {OT }}}$ and $\Delta \mathrm{V}_{\text {th } N_{\text {IT }}}$ separately, for $\mathrm{ReS}_{2}$ and MoS $\mathrm{S}_{2}$, leading to a quicker (lower $\mathrm{T}$ ) transition from CW to ACW hysteresis for ReS2. (d) Evolution of average CPD values for $\mathrm{ReS}_{2}$ and $\mathrm{MoS}_{2}$ with increasing T.

Finally, T-dependent KPFM measurements were performed to confirm the proposed model. The average contact potential difference (CPD) measured for $\mathrm{ReS}_{2}$ and $\mathrm{MoS}_{2}$ is shown in Fig. 4(d). We define CPD by

$$
\phi_{\text {sample }}=\phi_{\text {tip }}-[q \times C P D],
$$

where $\phi_{\text {sample }}$ and $\phi_{\text {tip }}$ represent the work function of the sample and the tip, respectively, and $\mathrm{q}$ is the electronic charge. The CPD values obtained are consistent with previous reports. ${ }^{29,30} \mathrm{CPD}$ values for $\mathrm{ReS}_{2}$ increase with increasing $\mathrm{T}$, whereas they remain almost unchanged for $\mathrm{MoS}_{2}$. The temperatures for crossover from CW to ACW hysteresis are marked as $\mathrm{T}_{\mathrm{CO}, \mathrm{ReS}_{2}}$ and $\mathrm{T}_{\mathrm{CO}, \mathrm{MoS}_{2}}$. The material with a more negative CPD value implies a larger work function and more $\mathrm{e}^{-}$depletion [Eq. (2)]. According to previous reports, gas adsorbates that act as $\mathrm{e}^{-}$acceptors are responsible for $\mathrm{e}^{-}$depletion, leading to more negative CPD. ${ }^{30-32}$ However, all the measurements in this work are carried out in a controlled nitrogen ambient, ruling out the presence of adsorbates as a possible cause for $\mathrm{e}^{-}$depletion. Therefore, we infer intrinsic trapping as the likely reason for $\mathrm{e}^{-}$ depletion, resulting in more negative CPD values at RT for $\mathrm{ReS}_{2}$. Moreover, intrinsic trapping can only be observed in devices with fully depleted channels; ${ }^{2,26}$ hence, at higher $\mathrm{T}$, the effect of intrinsic traps is nullified and oxide trapping dominates. As shown in Fig. 4(d), $\mathrm{T}_{\mathrm{CO}, \mathrm{ReS}_{2}}$ is less than $\mathrm{T}_{\mathrm{CO}, \mathrm{MoS}_{2}}$, implying that the effect of intrinsic traps persists for much higher T in $\mathrm{MoS}_{2}$ than for $\mathrm{ReS}_{2}$ consistent with the proposed model. The CPD maps for $\mathrm{ReS}_{2}$ are shown in Fig. 5(a) at $323 \mathrm{~K}$ and Fig. 5(b) at $373 \mathrm{~K}$ and for $\mathrm{MoS}_{2}$ in Fig. 5(c) at $323 \mathrm{~K}$ and Fig. $5(\mathrm{~d})$ at $373 \mathrm{~K}$. The contrast for all the images is adjusted on the same scale, clearly showing the most negative CPD for $\mathrm{ReS}_{2}$ at RT in Fig. 5(a).

To conclude, in this report, we demonstrate thermally assisted memory using back-gated vdP FETs with few-layer $\mathrm{ReS}_{2}$ and $\mathrm{MoS}_{2}$ as (a)

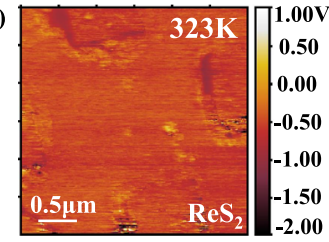

(c)

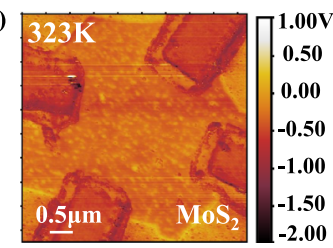

(b)

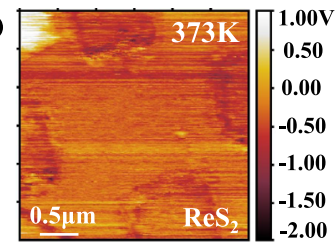

(d)

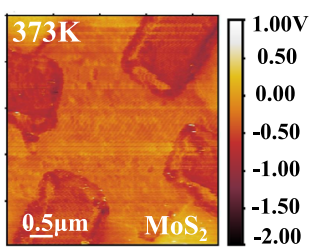

FIG. 5. CPD maps for $\mathrm{ReS}_{2}$ at (a) $323 \mathrm{~K}$ and (b) $373 \mathrm{~K}$ and for $\mathrm{MoS}_{2}$ at (c) $323 \mathrm{~K}$ and (d) $373 \mathrm{~K}$. The contrast for all the CPDs is adjusted on the same scale.

the channel materials. The transfer characteristics show a change in the hysteresis direction from $\mathrm{CW}$ to ACW with increasing $\mathrm{T}$, along with step-like jumps in the transfer curves at higher T (STC crossover hysteresis). Memory parameters such as RST-to-WR and RD windows are compared for memory operation. The step-like jumps in the transfer curve occur at much lower $\mathrm{T}$ for $\mathrm{ReS}_{2}(373 \mathrm{~K})$ as compared to $\mathrm{MoS}_{2}(400 \mathrm{~K})$, making it a better choice for memory applications. These results are ascribed to a combined effect of intrinsic traps at lower $\mathrm{T}$ and screening of gate voltage due to electron injection from the gate into oxide trapping sites at higher T. This physical model is corroborated through T-dependent KPFM measurements that show an increase in CPD for $\mathrm{ReS}_{2}$, while an almost constant CPD for $\mathrm{MoS}_{2}$ with increasing $\mathrm{T}$. This implies enhanced depletion of electrons in $\mathrm{ReS}_{2}$ with increasing $\mathrm{T}$, reinforcing the model of faster de-trapping of intrinsic $\mathrm{ReS}_{2}$ traps with $\mathrm{T}$ and hence a lower crossover $\mathrm{T}$.

See the supplementary material for the transconductance $\left(\mathrm{g}_{m}\right)$ vs gate voltage $\left(V_{G S}\right)$ plots at varying temperatures for $\mathrm{ReS}_{2}$ and $\mathrm{MoS}_{2}$ showing jumps during forward sweep and reverse sweep at high temperatures.

\section{REFERENCES}

${ }^{1}$ G. He, H. Ramamoorthy, C.-P. Kwan, Y.-H. Lee, J. Nathawat, R. Somphonsane, M. Matsunaga, A. Higuchi, T. Yamanaka, N. Aoki et al., "Thermally assisted nonvolatile memory in monolayer $\mathrm{MoS}_{2}$ transistors," Nano Lett. 16, 6445-6451 (2016).

${ }^{2}$ N. Kaushik, D. M. Mackenzie, K. Thakar, N. Goyal, B. Mukherjee, P. Boggild, D. H. Petersen, and S. Lodha, "Reversible hysteresis inversion in $\mathrm{MoS}_{2}$ field effect transistors," npj 2D Mater. Appl. 1, 34 (2017).

${ }^{3}$ M. Chen, H. Nam, S. Wi, G. Priessnitz, I. M. Gunawan, and X. Liang, "Multibit data storage states formed in plasma-treated $\mathrm{MoS}_{2}$ transistors," ACS Nano 8, 4023-4032 (2014).

${ }^{4}$ S. Bertolazzi, D. Krasnozhon, and A. Kis, "Nonvolatile memory cells based on $\mathrm{MoS}_{2}$ /graphene heterostructures," ACS Nano 7, 3246-3252 (2013).

${ }^{5}$ M. S. Choi, G.-H. Lee, Y.-J. Yu, D.-Y. Lee, S. H. Lee, P. Kim, J. Hone, and W. J. Yoo, "Controlled charge trapping by molybdenum disulphide and graphene in ultrathin heterostructured memory devices," Nat. Commun. 4, 1624 (2013).

${ }^{6}$ V. K. Sangwan, D. Jariwala, I. S. Kim, K.-S. Chen, T. J. Marks, L. J. Lauhon, and M. C. Hersam, "Gate-tunable memristive phenomena mediated by grain boundaries in single-layer $\mathrm{MoS}_{2}$," Nat. Nanotechnol. 10, 403 (2015). 
${ }^{7}$ Q. H. Wang, K. Kalantar-Zadeh, A. Kis, J. N. Coleman, and M. S. Strano, "Electronics and optoelectronics of two-dimensional transition metal dichalcogenides," Nat. Nanotechnol. 7, 699 (2012).

${ }^{8}$ Y. Yoon, K. Ganapathi, and S. Salahuddin, "How good can monolayer $\mathrm{MoS}_{2}$ transistors be?," Nano Lett. 11, 3768-3773 (2011).

${ }^{9}$ H. Wang, L. Yu, Y.-H. Lee, Y. Shi, A. Hsu, M. L. Chin, L.-J. Li, M. Dubey, J. Kong, and T. Palacios, "Integrated circuits based on bilayer $\mathrm{MoS}_{2}$ transistors," Nano Lett. 12, 4674-4680 (2012).

${ }^{10}$ E. Zhang, Y. Jin, X. Yuan, W. Wang, C. Zhang, L. Tang, S. Liu, P. Zhou, W. $\mathrm{Hu}$, and F. Xiu, "ReS ${ }_{2}$-based field-effect transistors and photodetectors," Adv. Funct. Mater. 25, 4076-4082 (2015).

${ }^{11}$ Y. Xiong, H. Chen, D. W. Zhang, and P. Zhou, "Electronic and optoelectronic applications based on $\mathrm{ReS}_{2}$," Phys. Status Solidi RRL 13, 1800658 (2019).

${ }^{12}$ S. Tongay, H. Sahin, C. Ko, A. Luce, W. Fan, K. Liu, J. Zhou, Y.-S. Huang, C.H. Ho, J. Yan et al., "Monolayer behaviour in bulk $\mathrm{ReS}_{2}$ due to electronic and vibrational decoupling," Nat. Commun. 5, 3252 (2014).

${ }^{13}$ E. Liu, M. Long, J. Zeng, W. Luo, Y. Wang, Y. Pan, W. Zhou, B. Wang, W. Hu, Z. Ni et al., "High responsivity phototransistors based on few-layer $\mathrm{ReS}_{2}$ for weak signal detection,” Adv. Funct. Mater. 26, 1938-1944 (2016).

${ }^{14}$ D. K. Schroder and J. A. Babcock, "Negative bias temperature instability: Road to cross in deep submicron silicon semiconductor manufacturing," J. Appl. Phys. 94, 1-18 (2003).

${ }^{15} \mathrm{~S}$. Hong, O. Auciello, and D. Wouters, Emerging Non-Volatile Memories (Springer, 2014).

${ }^{16}$ M. K. Patterson, "The effect of data center temperature on energy efficiency," in 2008 11th Intersociety Conference on Thermal and Thermomechanical Phenomena in Electronic Systems (IEEE, 2008), pp. 1167-1174.

${ }^{17}$ N. El-Sayed, I. A. Stefanovici, G. Amvrosiadis, A. A. Hwang, and B. Schroeder, "Temperature management in data centers: Why some (might) like it hot," in Proceedings of the ACM Sigmetrics Performance Evaluation Review (2012), Vol. 40, pp. 163-174.

${ }^{18}$ D. M. Mackenzie, J. D. Buron, P. R. Whelan, J. M. Caridad, M. Bjergfelt, B. Luo, A. Shivayogimath, A. L. Smitshuysen, J. D. Thomsen, T. J. Booth et al., "Quality assessment of graphene: Continuity, uniformity, and accuracy of mobility measurements," Nano Res. 10, 3596-3605 (2017).

${ }^{19}$ N. Goyal, N. Kaushik, H. Jawa, and S. Lodha, "Enhanced stability and performance of few-layer black phosphorus transistors by electron beam irradiation," Nanoscale 10, 11616-11623 (2018).

${ }^{20}$ N. Goyal, N. Parihar, H. Jawa, S. Mahapatra, and S. Lodha, "Accurate threshold voltage reliability evaluation of thin $\mathrm{Al}_{2} \mathrm{O}_{3}$ top gate dielectric black phosphorous FETs using ultrafast measurement pulses," ACS Appl. Mater. Interfaces 11, 23673-23680 (2019).

${ }^{21}$ A. D. Bartolomeo, L. Genovese, F. Giubileo, L. Iemmo, G. Luongo, T. Foller, and M. Schleberger, "Hysteresis in the transfer characteristics of $\mathrm{MoS}_{2}$ transistors," 2D Mater. 5, 015014 (2017).

${ }^{22}$ Y. Park, H. W. Baac, J. Heo, and G. Yoo, "Thermally activated trap charges responsible for hysteresis in multilayer $\mathrm{MoS}_{2}$ field-effect transistors," Appl. Phys. Lett. 108, 083102 (2016).

${ }^{23}$ A. K. Geim and K. S. Novoselov, "The rise of graphene," in Nanoscience and Technology: A Collection of Reviews from Nature Journals (World Scientific, 2010), pp. 11-19.

${ }^{24}$ A. K. Geim, "Nobel lecture: Random walk to graphene," Rev. Mod. Phys. 83, $851(2011)$.

${ }^{25}$ N. Goyal, D. M. Mackenzie, H. Jawa, D. H. Petersen, and S. Lodha, “Thermally aided nonvolatile memory using $\mathrm{ReS}_{2}$ transistors," in 76th Device Research Conference (IEEE, 2018).

${ }^{26}$ H.-C. Lin, C.-H. Hung, W.-C. Chen, Z.-M. Lin, H.-H. Hsu, and T.-Y. Hunag, "Origin of hysteresis in current-voltage characteristics of polycrystalline silicon thin-film transistors," J. Appl. Phys. 105, 054502 (2009).

${ }^{27}$ N. Kaushik, S. Ghosh, and S. Lodha, "Low-frequency noise in supported and suspended $\mathrm{MoS}_{2}$ transistors," IEEE Trans. Electron Devices 65, 4135-4140 (2018).

${ }^{28}$ K. Thakar, B. Mukherjee, S. Grover, N. Kaushik, M. Deshmukh, and S. Lodha, "Multilayer $\mathrm{ReS}_{2}$ photodetectors with gate tunability for high responsivity and high-speed applications," ACS Appl. Mater. Interfaces 10, 36512-36522 (2018).

${ }^{29}$ F. Li, J. Qi, M. Xu, J. Xiao, Y. Xu, X. Zhang, S. Liu, and Y. Zhang, "Layer dependence and light tuning surface potential of $2 \mathrm{~d} \mathrm{MoS}$ on various substrates," Small 13, 1603103 (2017).

${ }^{30}$ J. H. Kim, J. Lee, J. H. Kim, C. Hwang, C. Lee, and J. Y. Park, "Work function variation of $\mathrm{MoS}_{2}$ atomic layers grown with chemical vapor deposition: The effects of thickness and the adsorption of water/oxygen molecules," Appl. Phys. Lett. 106, 251606 (2015).

${ }^{31}$ S. Y. Lee, U. J. Kim, J. Chung, H. Nam, H. Y. Jeong, G. H. Han, H. Kim, H. M. Oh, H. Lee, H. Kim et al., "Large work function modulation of monolayer $\mathrm{MoS}_{2}$ by ambient gases," ACS Nano 10, 6100-6107 (2016).

${ }^{32}$ Y. Feng, K. Zhang, H. Li, F. Wang, B. Zhou, M. Fang, W. Wang, J. Wei, and H. P. Wong, "In situ visualization and detection of surface potential variation of mono and multilayer $\mathrm{MoS}_{2}$ under different humidities using kelvin probe force microscopy," Nanotechnology 28, 295705 (2017). 